\title{
SYSTEM DESIGN CANVAS FOR IDENTIFYING LEVERAGE POINTS IN COMPLEX SYSTEMS: A CASE STUDY OF THE AGRICULTURAL SYSTEM MODELS, CAMBODIA
}

\author{
Komaki, Akinori; \\ Kodaka, Akira; \\ Nakamura, Eri; \\ Ohno, Yu; \\ Kohtake, Naohiko \\ Graduate School of System Design and Management, Keio University
}

\begin{abstract}
There has been a growing recognition that a systems thinking approach, which pays attention to the entire dynamic system, its constituent parts and the interactions among those parts, is an effective approach for tackling complex issues that exhibit nonlinear behaviour and involve multiple stakeholders. To understand a complex system, a systems thinking approach must identify the leverage points, which are non-intuitive and difficult to identify. This paper proposes a design tool called System Design Canvas, which supports the identification of system variables and leverage points from technological sensing data and human data while understanding stakeholders' mental model. The present case study confirms that System Design Canvas can identify system variables and leverage points that are required for the current agricultural system in Cambodia.
\end{abstract}

Keywords: Design methods, Design methodology, Design process, Design Canvas, Systems Design

Contact:

Komaki, Akinori

Keio University

Graduate School of System Design and Management

Japan

sun.stone.mind@gmail.com

Cite this article: Komaki, A., Kodaka, A., Nakamura, E., Ohno, Y., Kohtake, N. (2021) 'System Design Canvas for Identifying Leverage Points in Complex Systems: A Case Study of the Agricultural System Models, Cambodia', in Proceedings of the International Conference on Engineering Design (ICED21), Gothenburg, Sweden, 16-20 August 2021. DOI:10.1017/pds.2021.551 


\section{INTRODUCTION}

Notwithstanding many trials to solve complex issues faced by modern societies, solutions to such issues are seldom long lasting because we usually employ the traditional linear thinking approach. Linear thinking appears natural and simple; however, it does not usually lead us to long-term solutions in the context of highly complex and multi-dimensional societies. For example, chronic conflict among stakeholders lasts in sustainability of natural resource management. Therefore, it has been increasingly recognised that the systems thinking approach, which considers the whole dynamic system along with its constituent parts and their interactions, is effective for handling complex issues. Systems thinking is a fundamentally interdisciplinary field of study that helps us understand complex systems (Sterman, 2001). It helps us comprehend systemic structure and the human mental model using a causal loop diagram as the main analytical tool. Moreover, it contributes to enable the identification and visualisation of points which enable to create a big impact for solving issues in the complex system with small efforts. It is called 'leverage points', which are potential intervention points.

To understand a complex system, we must identify the leverage points, which exist in all systems. However, the leverage points are non-intuitive and difficult to identify (Meadows, 2008). When we face difficulty in determining the leverage points and identifying the parts affected by the intervention, the system variable level in the causal loop diagram should be considered. When we create a causal loop diagram, we do not intend to capture the whole reality in one diagram. Instead, we want to map a part of that reality in such a way that it provides us a basic understanding of the entire complex system. When we are observing a complex system that contains an endless number of system variables, the system must be simplified and the system boundaries must be defined within a determined spatiotemporal scale.

This paper aims to support application of systems thinking for better understanding of complex issues by identifying system variables and leverage points. To that end, System Design Canvas is proposed. It is a design tool that supports the identification of system variables and leverage points from technological sensing data and human data while also understanding stakeholders' mental model. System Design Canvas guides the systems thinking processes of system engineers, system designers and practitioners. To demonstrate the preliminary identification of system variables and subsequent leverage points using System Design Canvas, we apply the tool in a case study of the current Cambodian agricultural system, which is a complex mix of microfinance and farming issues. To understand the complexity of this system, we collected mental models of the key stakeholders and the challenges faced by the agricultural sectors in Cambodia.

\section{IDENTIFYING LEVERAGE POINT USING SYSTEMS THINKING}

Systems thinking has gained popularity as a 'new way of thinking' to understand and manage complex problems (Bosch et al., 2015), In particular, it detects whether the extent of a complex problem is local or global (Bosch et al., 2007; Cabrera et al., 2008). Systems thinking captures the causal relationships and feedback flows from a high viewpoint in a causal loop diagram. Systems thinking is applicable in various fields and systems such as economics (Arthur, 1994), decision making (Maani and Maharraj, 2004) and practice of sustainability (Nguyen et al., 2013).

Once a representative causal loop diagram of a system has been constructed and validated, the leverage points of its complex and persistent problems become more apparent (Maani and Cavana, 2007). Leverage points are "places in complex systems where a small shift in one thing can produce big changes in everything' (Meadows, 2008). Meadows identified twelve types of leverage points, ranging from shallow to deep. At shallow leverage points, interventions can be relatively easily implemented without changing the overall functioning of the system. At deep leverage points, implementing the interventions is more difficult and risks a transformational change. However, interventions at deep leverage points can lead to long-lasting and significant improvements (Salisbury, 1996).

To explore the shallow/deep leverage points, one must identify the system archetypes, or common behavioural patterns of the system (Senge, 2006). Approximately one dozen systems archetypes have been classified, nine of which were applied in Senge's paper; this study applied those classifications. A deep understanding of the causal loop diagram enables us to know stakeholders' mental model. This also reveals areas of leverage points which have a large influence on the holistic system through a small action. The systems-based Evolutionary Learning Laboratory (ELLab) uses participatory systems analysis to understand the stakeholders' mental model and identify the leverage points of systemic 
interventions (Bosch et al., 2014). ELLab is a seven-step iterative process of group thinking and acting, in which the participants engage in well-defined activities. Through these activities, the participants collectively solve complex multi-dimensional and multi-stakeholder problems in an experimental laboratory environment. ELLab has been applied in many research cases (Banson et al., 2015).

\section{SYSTEM DESIGN CANVAS}

This paper proposes System Design Canvas (Figure 1), a design tool that supports the identification of system variables, the construction of causal loop diagrams from sensing and human data and recognising leverage points of systems. System Design Canvas guides systems thinking process while enhancing quality of system variables and can help system engineers, system designers and stakeholders to reach a consensus on the intervention points of a complex system. The canvas is selfexplanatory and requires no significant training efforts by system engineers and system designers for its application. The canvas is simply a one-page graphic structure that enables an immediate current system overview using key system variables, a causal loop diagram and the leverage points. We designed a three-step protocol in order to fill in the canvas as follows:

- $\quad$ STEP 1: Decide Research Purpose and System Boundaries

Before starting systems thinking with this canvas, the research goal and hypothesis related to target topics are clarified. Next, users investigate the holistic stakeholder relations to identify the key issue holders and involve people who should have ownership of this research. Then, they define system boundaries to focus on systemic resolution based on consensus building with key issue holders. System boundaries describe the system scope from the spatiotemporal perspectives of key stakeholders. On the canvas, these motivate the creation of three areas: 'Research Purpose and Question', 'Stakeholders' Profile (Role and Responsibility)' and 'System Boundaries'. They can iteratively fill the canvas in any order, but the most desirable process would start from 'Research Purpose \& Question' to refine your research question of your research.

- $\quad$ STEP 2: Gather System Variables by a 2 x 2 matrix

After STEP1, users investigate the key system variables by delving into the cultural/mental model using technological sensing data and human data. Generally, they go to fieldwork several times to observe stakeholder relations and understand key issue holders with an awareness of the presence of system boundaries. On the canvas, these motivate the creation of a $2 \times 2$ matrix area.

The uniqueness of this canvas feature lies in this $2 \times 2$ matrix area. This area can be described by a $2 \times 2$ matrix based on physical events, cultural and mental events, and sensing and human data (see Figure 1). The systems thinking approach embraces four levels of thinking (Maani And Cavana, 2007). This approach focuses on not only physical events which represent the most visible level of reality but also cultural and mental events which reflect the deepest and most profound assumptions. The vertical line on this canvas separates physical events from cultural and mental events. The horizontal rows show the difference between the input sources of the sensing and human data (interview). For example, the visible and characteristic system variables derived from technological measurements using devices such as smartphones and location-tracking devices are described in the upper-left column. Meanwhile, the visible system variables obtained through human interactions such as interviews and meetings are written in the upper-right column. Next, the canvas user investigates the reason why the visible level of system variables is derived from technological sensing data, recognizes patterns in which a larger set of data are linked together to create a history, and explores the cultural structure and stakeholders' mental model behind those patterns. Then, cultural and mental system variables are identified and filled in the bottom-left column. In the same way, the user investigates the reason why the visible system variables are obtained through human interactions, identifies cultural and mental system variables, and fills them in the bottom-right column.

- $\quad$ STEP 3: Analyse Causal Loops and Leverage Points

After STEP2, users visualise the causal relationships among the key system variables. They refer the key system variables in a $2 \times 2$ matrix as nodes and create connection between the two variables while repeating discussion and fieldwork to reach a consensus of causality with stakeholders. And they elucidate the system archetypes and find the leverage points with paying attention to the system variables with many causal inflows and outflows. On the canvas, these motivate the creation of three areas: 'Causal Loop Diagram', 'System Archetype' and 'Leverage Points'. 
The motivation of this work is the demonstration of System Design Canvas in a case study of the current agricultural system in Cambodia. An example case of Cambodian agricultural system is shown in Figure 1. The system focuses on exploring the recent agricultural challenges from farm and financial perspectives, to effectively understand the complex issues in Cambodia. This clarifies difficulties in Cambodian agriculture by gathering the mental models of stakeholders and provides a way for decision makers to anticipate the long-term consequences of their decisions and actions.

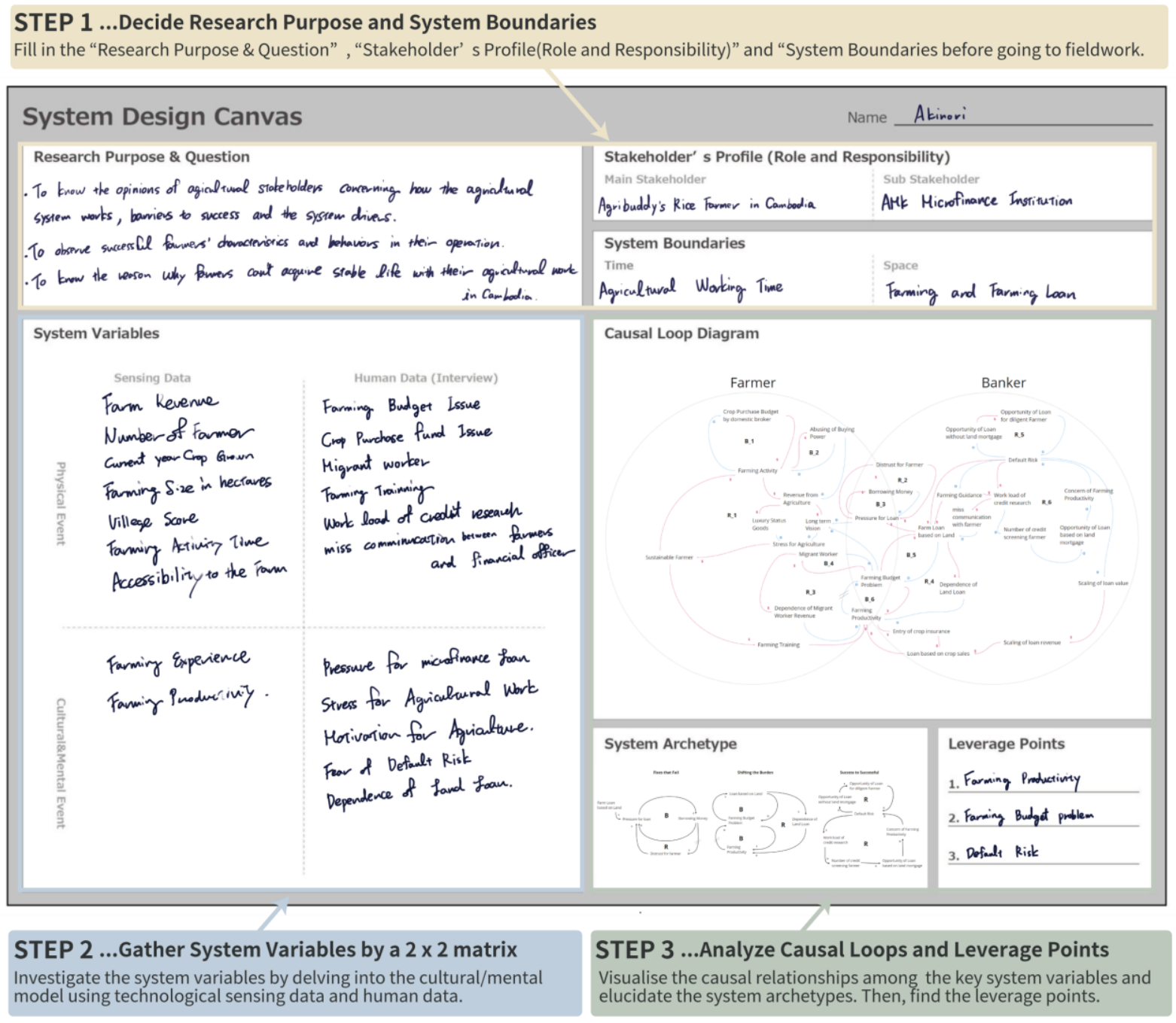

Figure 1. System Design Canvas

\section{APPLICATION OF SYSTEM DESIGN CANVAS TO THE CAMBODIAN AGRICULTURE STUDY}

We apply System Design Canvas in a case study of the current agribusiness in Cambodia to clarify the use of the Canvas and describe how to use the canvas. System Design Canvas unravelled the important dynamics of a complex system.

In the following sections, the research steps are described based on System Design Canvas to identify system variables and leverage points that are required for the current agricultural system in Cambodia. We collaborated with Agribuddy Ltd. (https://www.agribuddy.com/?lang=en), a company that offers a mobile online agricultural platform in Cambodia and other parts of Asia.

\subsection{Step1 for deciding research purpose and system boundaries}

Before travelling to Cambodia, we have conducted a desktop research of Cambodian agriculture. For example, Cambodian agriculture currently faces very complex farming and microfinance issues. Rice is the most important agricultural product in Cambodia, and the paddy yield has rapidly increased. More than 2.6 million Cambodian people have availed of microfinance loans, and many farmers have 
used such loans to improve their farming methods. However, the financial services offered by microfinance institutions (MFIs) are inaccessible to farmers with limited resources or collateral. Vulnerable farmers with limited resources cannot invest in their agricultural activities, which diminishes their farming output. After delving into the background of Cambodia agriculture from the desktop research, we filled in three areas of canvas ('Research Purpose and Question', 'Stakeholders' Profile (Role and Responsibility)' and 'System Boundaries').

\subsubsection{Research purpose and question}

We discussed the research purposes among our team members to define hypotheses on key issues and challenges faced by Cambodian farmers. The initial research purposes were as follows:

- Identify the agricultural stakeholders and acquire their opinions on how the agricultural system works, their barriers to success and the system drivers.

- To observe the characteristics and behaviours of successful farmers in their operation.

- To understand the mental model of farmers and MFI officers in Cambodia.

\subsubsection{Stakeholders' profile (role and responsibility)}

'Stakeholders' refer to all people who have some stake in the research purposes (defined in section 5.2.1), such as farmers, bank staff, insurance staff, middlemen, consumers or domestic and overseas buyers. The present research mainly focuses on Cambodian rice farmers as a key stakeholder because rice farmers can significantly influence the agriculture sector in Cambodia. To acquire a deep agricultural insight, we targeted small rice farmers receiving services from Agribuddy, as this group would likely be influenced by financial officers who provide microfinance. Therefore, financial officers would be defined as a sub-key stakeholder. To deepen our understanding of financial officers, we targeted the staff at Angkor Mikroheranhvatho Kampuchea (AMK) (https://www.amkcambodia. com/index.html), a registered MFI with headquarters in Phnom Penh, which collaborates with Agribuddy service.

\subsubsection{System boundaries}

All systems were defined by system boundaries. To understand a system properly, we need to define the system level we are observing, such as small transaction levels or global policy levels. Generally, the system boundaries are confined to a specified spatiotemporal scale (Haraldsson, 2004). In the present research, we closely observed the agricultural interaction between Agribuddy's rice farmers and the AMK staff offering the microfinance. The system boundaries were then defined by the working time of rice farmers, the farming area, the time of farming loan operations and places where farming loans are available.

\subsection{Step2 for gathering system variables by a $2 \times 2$ matrix}

We identified and refined the system variables through repeated fieldwork in the farming areas of Cambodia and deep discussions with stakeholders and filled in a $2 \times 2$ matrix area of the canvas. The system variables are mutually connected through causal relationships. Intuitive and countable system variables are preferred because when the system variables are excessive and uncountable, the system becomes intractably complex and uncertain. During our research, we were accompanied by a local guide working for Agribuddy. The fieldwork proceeded in three steps as outlined below:

- Acquiring qualitative human data from interviews

Fieldwork was conducted thrice in the farming areas around Siem Reap and in bank offices around Phnom Penh, Cambodia (Figure 2). Each fieldwork was conducted over one or two weeks in Cambodia. Our team member tried to understand the local lifestyle, food, local culture and empathise with Cambodian farmers' personality to gain insights into their mental model, which reflects their beliefs, values and assumptions that influence why things work the way they do in Cambodia. Over the total fieldwork period, we interviewed 22 small local farmers and 6 AMK staff members (one executive, one team manager and four branch officers), who gave their opinions. Cambodia has two seasons: a dry season and a rainy season. Farmers who can access a water source for irrigation grow rice twice a year. Many farmers lack sufficient funds, which compels them to negotiate with a local seller or bank officer to obtain farming inputs on credit. The money is repaid with interest when the farmers have harvested their crops. Local sellers and 
bankers have always lent money to long-term residents in the village. Newcomers to village are denied loans owing to the heavy workload of researching their credit ratings. Many young farmers seek work near cities such as Phnom Penh or near large overseas cities to stabilise their lifestyles. Many uneducated women and elderly people continue farming in the villages.

In this survey, 'Farming Budget Problem', 'Migrant Worker' and 'Work Load of Credit Research' were validated as the key system variables and were written in the upper-right column of the $2 \times 2$ matrix area. Insights into the cultural and mental events in Cambodia were provided by statements such as 'Pressure for Loan Payment', 'Fear of Default Risk', and 'Dependence of Land Loan'. Those were written in the bottom-right column of the $2 \times 2$ matrix area.

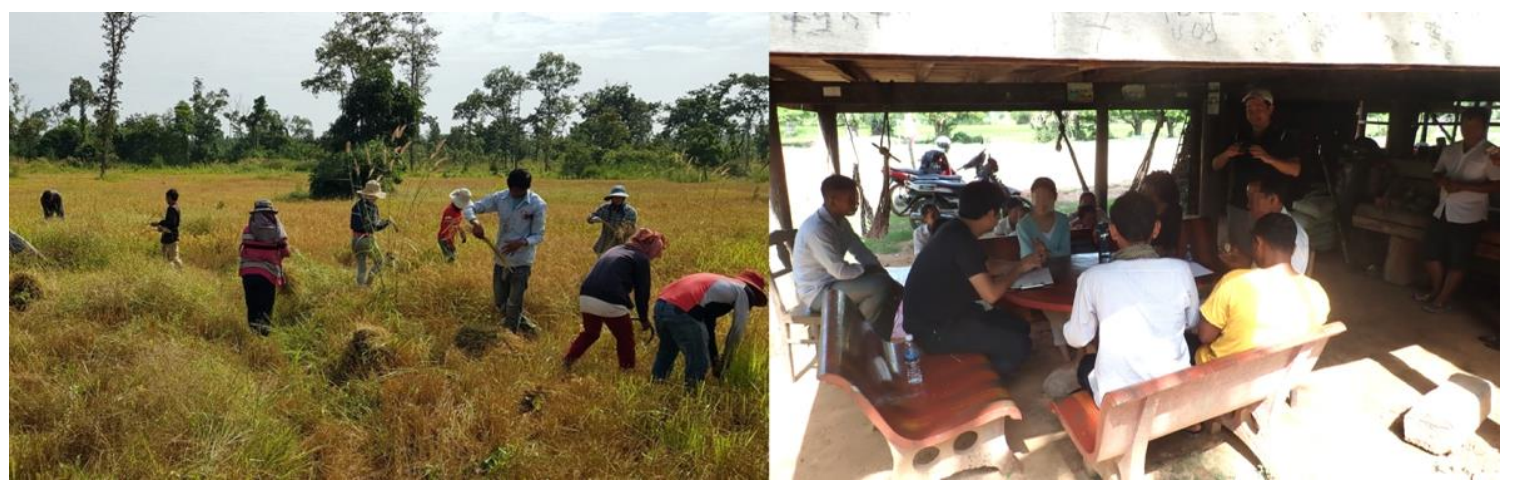

Figure 2. Fieldwork and interview

- $\quad$ Acquiring implicit opinions from LifeLog data

The human daily-life data from LifeLog provide the implicit opinions and motivations of the loggers. After the interview process in Siem Reap, each interviewee reported their activities during the past week on a LifeLog sheet (Figure 3) to review the past week. Cambodian farmers were given the option to write the LifeLog sheet in Khmer. Then, a local guide working for Agribuddy Ltd. has translated the same from Khmer to English. The farmers' LifeLog sheets revealed an observable lifestyle difference depending on the village. For example, some village famers lacking good access to farming areas spend most of their time travelling to their farm and meeting money lenders and bankers. Reclamation of farm area by lenders and bankers is a major fear among farmers. To avoid this situation, the farmers approach lenders and bankers to explain their agricultural situation. However, as farmers and bankers lead very different lifestyles, the scheduled meeting is often disrupted by miscommunication, leading to stress. It was also noted that residents in villages closer to the town are better able to access markets and training opportunities from non-governmental organisations than residents far from town. From the LifeLog data, we identified 'Miscommunication between Farmers and Financial Officers' as numerical physical event and was written in the upper-right column of the $2 \times 2$ matrix area. Furthermore, we recognized 'Stress for Agricultural Work' and 'Motivation for Agriculture' as the key cultural and mental events and were written in the bottom-right column of the $2 \times 2$ matrix area.

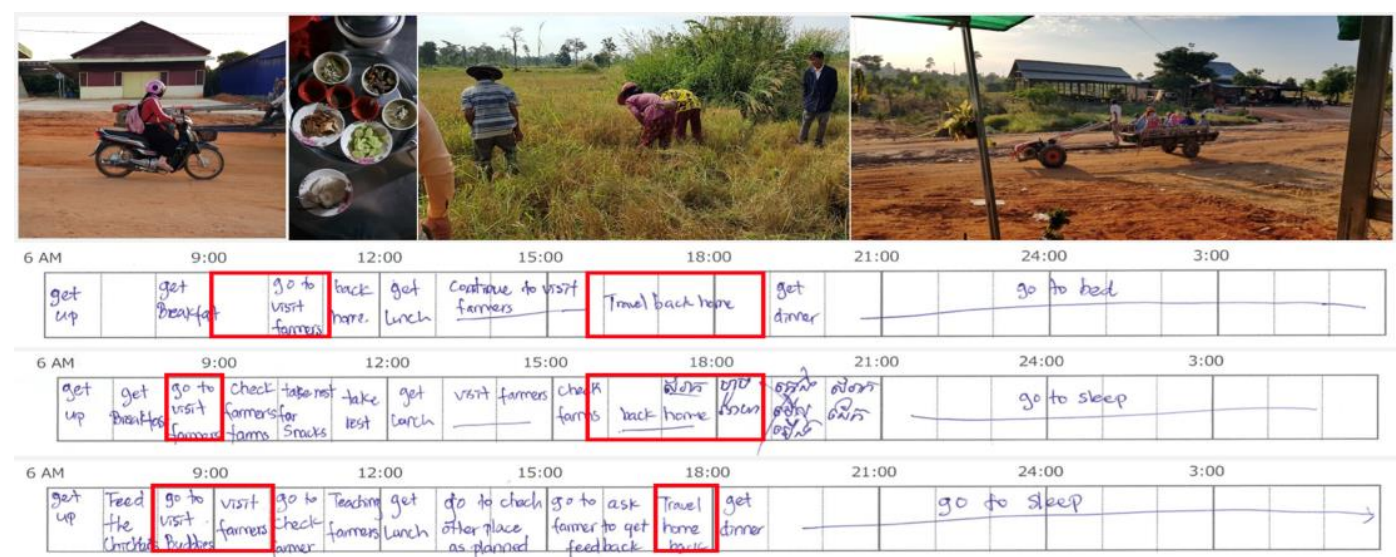

Figure 3. Representative LifeLog of a farmer

- $\quad$ Acquiring system variables from sensing data 
To identify the quantitative system variables, we investigated the Agribuddy's mobile platform data, the open data and the satellite data of the farmers' activity. Quantitative data are important for verifying the accuracy of qualitative data. Agribuddy Ltd. operates a mobile online agricultural platform that offers various services to farmers and other players in the agricultural sector. A class of farmers in Agribuddy, referred to as 'Buddies', directly report their local farming activities on the mobile platform. System variables such as 'Farmer's revenue', 'Number of farmers', 'Distance from home to farm', and 'Crop Purchase Fund Issue' were identified as numerical physical events and were written in the upper-left column of the 2 x 2 matrix area. Meanwhile, 'Farming Experience' and 'Farming Productivity' were identified as the key cultural and mental events and were written in the bottom-left column of the $2 \times 2$ matrix area. For example, open data (https://opendevelopmentcambodia.net/) revealed that Cambodia's rice farming area is approximately 3.3 million hectares, with the yield per hectare being 3.5 tonnes; and middlemen pay 200 US dollars per tonne of rice, which means that a crop purchase fund of around 2.3 billion US dollars would be needed. However, the loan size of the Agricultural and Rural Development Bank (which issues government loans) is only around 50 million US dollars. Therefore, we numerically confirmed that crop purchase funds are limited.

\subsection{Step3 for analysing causal loops and leverage points}

After Step2, we developed the causal loop diagram of the current agribusiness in Cambodia to find the leverage points using System Design Canvas. The causal loop diagram mainly focuses on exploring recent agricultural challenges from the farm and financial sides and effectively understand the complex issues in Cambodia. We filled in three areas of canvas ('Causal Loop Diagram', 'System Archetype' and 'Leverage Points').

\subsubsection{Constructing a causal loop diagram}

To understand the current Cambodian agricultural system and its inflows and outflows, we constructed a causal loop diagram, which explains the reality through causalities between the system variables and their dynamic circular influences. Each pair of variables can move in the same (s) or opposite (o) direction. Generally, a causal loop expresses reinforcing loops (positive feedback) or balancing loops (negative feedback).

Figure 4 illustrates the causal loop diagram of the current Cambodian agricultural system. At the left and right sides are the causal loops of the rice farmers and bankers, respectively. The current Cambodian agricultural system was closely observed with the above system variables and the repeated patterns that can predict the core behaviour in the problem were identified. Our team promoted remote meetings through online tools and engaged in repeated discussion with Agribuddy's team to obtain a consensus about the causality. Accordingly, the causal loop diagram of the current agricultural system was constructed with six reinforcing loops (R) and six balancing loops (B).

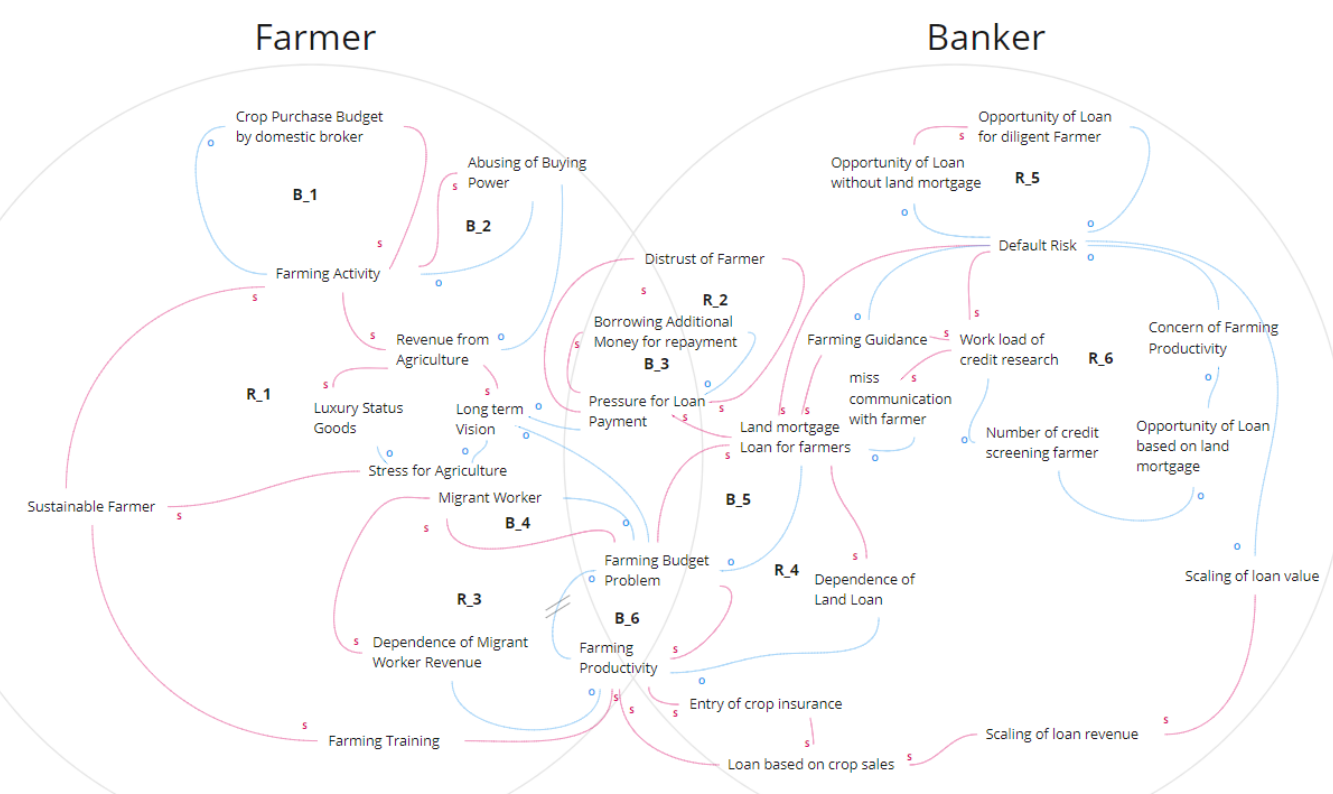

Figure 4. Causal loop diagram of the current agricultural system in Cambodia 


\subsubsection{System archetypes and candidates of leverage points}

The twelve archetypes developed in the domain of systems engineering (Senge, 2006) were considered comprehensively to facilitate discussion among the key stakeholders, and to have mutual understanding on the target issue. Eventually, it was consented among them that the causal loop diagram of the Cambodian agricultural system contains three dominant system archetypes: 'Fixes that Fail', 'Shifting the Burden' and 'Success to Successful'. These generic systems models or templates represent a wide range of situations and provide a high-level map of dynamic processes (Maani and Cavana, 2007).

The 'Fixes that Fail' archetype describes a situation in which a fix that is effective in the short term creates side effects that affect the long-term behaviour of the system and may result in the need for more fixes. This archetype comprises two feedback loops: the balancing feedback loop of the corrective action and the reinforcing feedback loop of the unintended consequences. As shown in Figure 5, when farmers feel the pressure for taking a microfinance loan in Cambodia, they easily seek another loan to reduce their short-term debt. An unintended consequence of this solution is the increased mistrust of farmers by local bankers. This mistrust negatively affects the financial credit of farmers and increases the pressure to borrow over the long term.

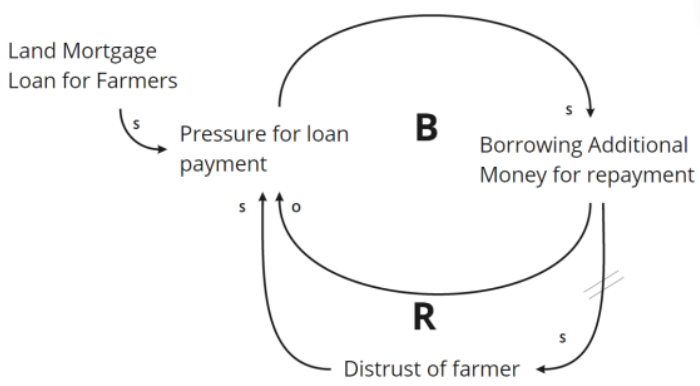

Figure 5. 'Fixes that Fail' system archetype in Cambodian agricultural system

The 'Shifting the Burden' archetype shows how quick-fix solutions create further dependence on symptomatic solutions. Symptomatic solutions divert attention from the fundamental problems. This archetype comprises two balancing loops and one reinforcing loop. As shown in Figure 6, many Cambodian rice farmers face farming budget problems and cannot obtain sufficient farming inputs (such as fertilisers, pesticides and seeds) for their agriculture. These budget problems have received considerable land loan-based financial assistance from MFIs such as AMK. Although this quick-fix solution temporarily alleviates the pain of the budget problem, it imposes a high interest rate and encourages loan dependence. From a long-term perspective, farmers must increase their productivity and raise their farming profits. Therefore, loans themselves cannot address the fundamental problem of farming.

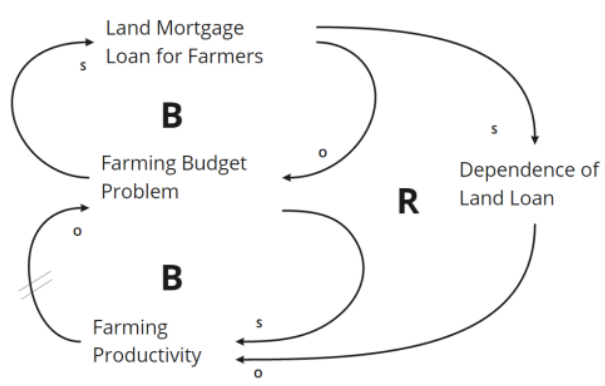

Figure 6. 'Shifting the burden' system archetype in cambodian agricultural system

The 'Success to Successful' archetype describes the phenomenon of 'the rich becoming richer and the poor becoming poorer'. This archetype comprises two reinforcing loops in delicate balance. In the Cambodia case study, this causal loop expresses the banker's mental model (Figure 7). Many microfinance providers are concerned about default and arrear risks when loaning to small rice farmers in Cambodia. Each credit officer is responsible for a limited number of farmers (around 100) and will preferentially lend to those with property or collateral. The opportunity for obtaining a loan without a land mortgage is reduced even if farmers are diligent. The default risk and decreasing opportunity of loans without land mortgages are serious problems in Cambodia. 


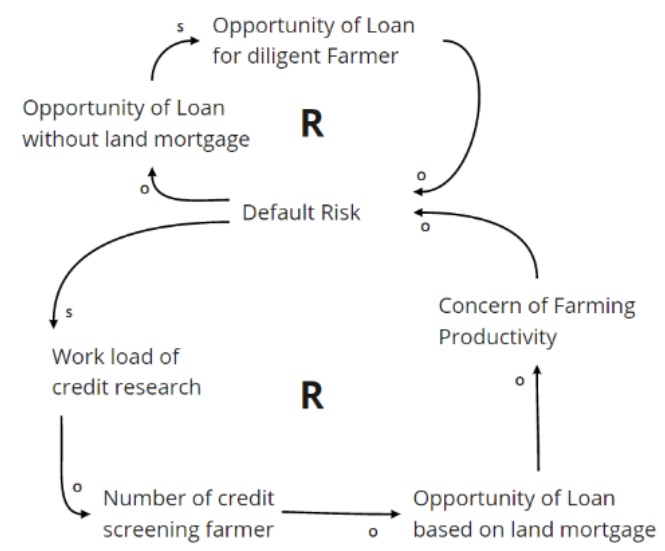

Figure 7. 'Success to Successful' system archetype in Cambodian agricultural system

At the leverage points within a complex system, a small shift can generate a large change in the overall system, potentially leading to significant lasting improvements. Therefore, leverage points are root causes of challenges. We repeatedly rewrote System Design Canvas to identify the leverage points that are useful for building consensus on agricultural issues with stakeholders. In the Cambodian agricultural system, after continuous discussion, the leverage points were identified as 'Farming Productivity', 'Farming Budget Problem' and 'Default Risk'. The case study is ongoing. As the next step, we will apply several small interventions to address these leverage points.

\section{DISCUSSION}

The System Design Canvas process was tested on system engineers and Agribuddy members over an approximate time course of one year in the Cambodian agricultural project. We have validated whether the canvas supports systems thinking from viewpoints of challenges people generally face during complex stakeholder requirement analysis. We gained three categories of insights as following:

\section{Quality guarantee of system variables}

System Design Canvas provides tips for improving the system variables using a $2 \times 2$ matrix based on physical events, cultural and mental events, sensing data and human data. When a beginner draws a causal loop diagram, it lacks consistency in terms of the level of system variables. The $2 \times 2$ matrix strengthens the quality of the system variables by incorporating quantitative sensing data and qualitative human data.

\section{Lead leverage points to supporting systems thinking}

This canvas is applicable to a wide range of systems thinking processes in business decision making, product and service market research and academic design research. In particular, when complex stakeholder requirement analysis is elicited, System Design Canvas provides a logical framework to unravel complex stakeholder needs and issues systematically. When used in the early system-design phase, this canvas provides a holistic perspective and accelerates decision making using leverage points.

\section{Enhancing users' communication}

During the current agricultural project, System Design Canvas smoothed the communication between the system engineer and business designer. We have discussed how to utilise this canvas for the project formulation of development agencies such as Japan International Cooperation Agency (JICA), and the discussion is ongoing. Positive reactions imply that System Design Canvas effectively provides an identification process of the leverage points to system engineers who are unfamiliar with the systems thinking process while promoting holistic and long-term perspective discussions among stakeholders. The canvas is also a useful educational tool for the staff of international organisations and governments for their policy formulation and program design. 


\section{CONCLUSION}

Increasingly complex problems and challenges require new and holistic ways of thinking for understanding the long-term consequences of a solution. This paper proposes a tool named System Design Canvas, on which users can analyse complex systems and identify the leverage points for improving a system in the future. This canvas guides the systems thinking procedure and assists the identification of the key system variables, causal loop diagram and leverage points through fieldwork interviews, LifeLog and quantitative sensing data. As a demonstration of its efficacy, System Design Canvas was applied to complex Cambodian agricultural systems. The development of System Design Canvas in the Cambodia case revealed key leverage points and highlighted the mental model of current small rice farmers and bankers. These individuals were mainly concerned with 'Farming Productivity', 'Farming Budget Problem' and 'Default Risk'. Through a year-long case study, System Design Canvas effectively promoted a consensus of the intervention points among stakeholders.

\section{ACKNOWLEDGEMENTS}

This work was supported by JSPS KAKENHI Grant Number JP19H04100. We would also like to acknowledge Agribuddy Ltd. Agribuddy members played an essential role in the fieldwork and analysing the design of the Cambodian agricultural system. Many conversations with the Agribuddy members also influenced the creation of System Design Canvas.

\section{REFERENCES}

Arthur, W. B. (1994). Increasing returns and path dependence in the economy, University of Michigan Press, Ann Arbor.

Banson, K. E., Nguyen, N. C., Bosch, O. J. and Nguyen, T. V. (2015), “A systems thinking approach to address the complexity of agribusiness for sustainable development in Africa: a case study in Ghana", Systems Research and Behavioral Science, Vol. 32 No. 6, pp. 672-688.

Bosch, O. J. H., King, C. A., Herbohn, J. L., Russell, I. W. and Smith, C. S. (2007), "Getting the big picture in natural resource management-systems thinking as 'method' for scientists, policy makers and other stakeholders", Systems Research and Behavioral Science: The Official Journal of the International Federation for Systems Research, Vol. 24 No. 2, pp. 217-232.

Bosch, O. and Nguyen, N. C. (2015), Systems thinking for everyone: The journey from theory to making an impact, Think2Impact Pty Ltd., Canberra, Australia.

Bosch, O. J. H., Nguyen, N. C. and Krishnamurthi, K. (2014), "Systems-based Evolutionary Learning Laboratories to enable Systemic Entrepreneurship in a Complex World (Invited Keynote Address)", In The 10th HSSS National \& International Conference: "Systemic Entrepreneurship-Innovations, Business, Growth”, Athens, 29-31 May 2014, The Hellenic Society for Systemic Studies (HSSS).

Cabrera, D., Colosi, L., and Lobdell, C. (2008), "Systems thinking", Evaluation and program planning, Vol. 31 No. 3, pp. 299-310.

Haraldsson, H. V. (2004), Introduction to system thinking and causal loop diagrams, Reports in Ecology and Environmental Engineering 2004, Vol 1, Lund University.

Maani, K. and Cavana, R. Y. (2007), Systems thinking, system dynamics: Managing change and complexity, Prentice Hall, Auckland, NZ.

Maani, K. E. and Maharaj, V. (2004), "Links between systems thinking and complex decision making. System Dynamics Review", Journal of the System Dynamics Society, Vol. 20 No. 1, pp. 21-48.

Meadows, D. H. (2008), Thinking in systems: A primer, chelsea green publishing, White River Junction, Vermont.

Nguyen, N. C., \& Bosch, O. J. (2013). “A systems thinking approach to identify leverage points for sustainability: a case study in the Cat Ba Biosphere Reserve, Vietnam", Systems Research and Behavioral Science, Vol. 30 No.2, pp. 104-115.

Salisbury, D. (1996), Five Technologies for Educational Change: Systems Thinking, Systems Design, Quality Science, Change Management, Instructional Technology, Educational Technology, New Jersey.

Senge, P. M. (2006), The fifth discipline: The art and practice of the learning organization (Rev. ed.), Currency Doubleday, New York.

Sterman, J. D. (2001), "System dynamics modeling: tools for learning in a complex world", California Management Review, Vol. 43 No. 4, pp. 8-25. 